VOL. 44 (1991) [239-244]

\title{
A NOTE ON STRONG MARKUŠEVIČ DECOMPOSITIONS OF BANACH SPACES
}

\author{
P.K. JaIn AND D.P. Sinha
}

The space $\ell^{\infty}$ is known to have no Schauder decomposition. It is proved here that $\ell^{\infty}$ does not even possess any strong Markuševič decomposition.

\section{INTRODUCTION}

A sequence $\left(G_{n}\right)$ of non-zero linear subspaces of a Banach space $E$ is said to be a decomposition of $E$ if for each $x \in E$ there exists a unique sequence $\left(x_{n}\right)$ with $x_{n} \in G_{n}(n=1,2, \ldots)$ such that the series $\sum_{n=1}^{\infty} x_{n}$ converges to $x$. This gives a unique sequence $\left(v_{n}\right)$ of linear projections on $E$ satisfying $v_{i} . v_{j}=0$, whenever $i \neq j$. If each $v_{n}$ is continuous then $\left(G_{n}\right)$ is said to be a Schauder decomposition. It is known that every infinite dimensional Banach space has a decomposition and that every decomposition may not be Schauder ([9], Theorem 1 and Example 2) so much so, that every Banach space need not possess a Schauder decomposition. In fact, the space $\ell^{\infty}$ does not possess any Schauder decomposition (Dean [2]). A detailed account of the theory of Schauder decompositions can be found in [10]. A more general concept than that of Schauder decompositions, namely Markuševič decompositions was introduced under the name "complete biorthogonal decompositions" in [1]. These decompositions together with a particular class of them, called the strong Markuševič decompositions, have been discussed in detail by the authors in $[5,6]$. It is natural to ask whether $\ell^{\infty}$ possesses a Markuševič decomposition. In the present note, we establish that $\ell^{\infty}$ does not have any strong Markuševič decomposition, countable or uncountable. The proof of our result is a little cumbersome and makes use of certain ideas developed by Lindenstrauss $[7]$.

\section{MaIn Result}

DEFINITION 1: An indexed collection $\left(G_{\lambda}\right)_{\lambda \in \Lambda}$ of non-zero closed linear subspaces of a Banach space $E$ is said to be a Markuševič decomposition ( $M$-decomposition)

Received 2nd October 1990

The research of the second author is supported by the CSIR (India).

Copyright Clearance Centre, Inc. Serial-fee code: 0004-9729/91 \$A2.00+0.00. 
of $E$ if there exist bounded linear projections $\left(v_{\lambda}\right)_{\lambda \in \Lambda}$ with $v_{\lambda}(E)=G_{\lambda}$ such that $\overline{\text { span }} \bigcup_{\lambda \in \Lambda} G_{\lambda}$ is dense in $E$ and $v_{\lambda}(x)=0(\lambda \in \Lambda)$, imply $x=0$.

It has been shown in [5] that the collection $\left(v_{\lambda}\right)_{\lambda \in \Lambda}$, called the associated family of coordinate projections, is uniquely determined by the $M$-decomposition and that every weakly compactly generated Banach space admits of a countable $M$-decomposition. Note that $\ell^{\infty}$ being the non-separable dual of a separable Banach space cannot be contained in any weakly compactly generated Banach space.

Definition 2: An $M$-decomposition $\left(G_{\lambda}\right)_{\lambda \in \Lambda}$ of a Banach space $E$ with the associated family of coordinate projections $\left(v_{\lambda}\right)_{\lambda \in \Lambda}$ is said to be a strong $M$-decomposition if each $x \in \overline{\operatorname{span}}_{\lambda \in \Lambda(x)} G_{\lambda}$, where $\Lambda(x)=\left\{\lambda \in \Lambda: v_{\lambda}(x) \neq 0\right\}$.

Clearly every Schauder decomposition is a countable strong $M$-decomposition. Every $M$-decomposition may not be a strong $M$-decomposition and a countable strong $M$-decomposition may not be a Schauder decomposition [6]. In the present discussion we shall write

$$
\sigma(x)=\left\{n: \alpha_{n} \neq 0\right\}, \quad\left(x=\left(\alpha_{n}\right) \in \ell^{\infty}\right) .
$$

THEOREM. The space $\ell^{\infty}$ has no countable or uncountable strong $M$-decomposition.

Proof: Write $E=\ell^{\infty}$. Let $\left(G_{\lambda}\right)_{\lambda \in \Lambda}$ be a strong $M$-decomposition of $E$ with the associated family of projections $\left(v_{\lambda}\right)_{\lambda \in \Lambda}$. Define seminorms on $E$ by

$$
t_{\lambda}(x)=\left\|v_{\lambda}\right\|^{-1}\left\|v_{\lambda}(x)\right\|, \quad(\lambda \in \Lambda) .
$$

Since $\left(v_{\lambda}\right)_{\lambda \in \Lambda}$ is total on $E$, the set

$$
(x, \varepsilon)=\left\{\lambda \in \Lambda: t_{\lambda}(x)>\varepsilon\right\}
$$

is finite for each $x \in E$ and $\varepsilon>0$. Without any loss of generality we may assume that $\mathbb{N}$ and $\Lambda$ are disjoint sets. Let $\Delta=\{0\} \cup \mathbb{N} \cup \Lambda$. Define a mapping $Q_{0}: E \rightarrow c_{0}(\Delta)$ by

$$
\left(Q_{0} x\right)(\delta)=\left\{\begin{array}{l}
2^{-n}(1+n)^{2}\|x\|, \quad(n=\delta / 2 \text { and } \delta=0,2,4 \ldots), \\
\alpha_{n} / n, \quad\left(n=(\delta+1) / 2, \quad \delta=1,3,5, \ldots \text { and } x=\left(\alpha_{i}\right)\right), \\
t_{\delta}(x), \quad(\delta \in \Lambda) .
\end{array}\right.
$$

Let $J$ denote the Day's locally uniformly convex norm as shown in [8] and $\|\cdot\|$, the usual sup norm on $c_{0}(\Delta)$. Then

$$
\|x\| / 2 \leqslant J(x) \leqslant\|x\| / \sqrt{3}, \quad\left(x \in c_{0}(\Delta)\right) .
$$


The function on $E$ given by

$$
\|\mid x\|_{0}=2 J\left(Q_{0} x\right)
$$

defines a norm on $E$ ([11], Lemma) and there is a $K>1$ such that

$$
\|x\| \leqslant\|\mid x\|_{0} \leqslant K\|x\|, \quad(x \in E) .
$$

Write $M=\sup \left\{\||x|\|_{0}:\|x\|=1\right\}$. Since $(3 M+1) / 4<M$, there is an $x_{1}=\left(\alpha_{i}^{(1)}\right) \epsilon$ $E$ with $\left\|x_{1}\right\|=1$ such that

$$
(3 M+1) / 4 \leqslant\left\|\mid x_{1}\right\|_{0} .
$$

We may assume that $\mathbb{N} \backslash \sigma\left(x_{1}\right)$ is infinite. Let $S_{1}$ be an infinite subset of $\mathbb{N} \backslash \sigma\left(x_{1}\right)$ such that $\mathbb{N} \backslash\left(\sigma(x) \cup S_{1}\right)$ is infinite and let $i_{1}$ be an integer in $\mathbb{N} \backslash\left(\sigma\left(x_{1}\right) \cup S_{1}\right)$. Writing

$$
\begin{aligned}
F_{1}= & \left\{y=\left(\beta_{i}\right) \in E:\|y\|=1, \quad\left|\beta_{i_{1}}\right|=1, \quad \beta_{i}=\alpha_{i}^{(1)},\right. \\
& \text { for all } \left.i \in \sigma(x) \cup S_{1} \text { and } \mathbb{N} \backslash\left(\sigma(y) \cup \sigma\left(x_{1}\right) \cup S_{1}\right) \text { is infinite }\right\}
\end{aligned}
$$

and $K_{1}=\sup \left\{\|y \mid\|_{0}: y \in F_{1}\right\}$ and since for each $y \in F_{1}, 2 x_{1}-y \in F_{1}$, we have $\left\|2 x_{1}\right\|-1 \leqslant K_{1}$. This gives

$$
K_{1}-1 \leqslant((4 K-3) M-1) / 2 K \leqslant(M-1) / 2 .
$$

Again, since $\left(3 K_{1}+1\right) / 4<K_{1}$, there is an $x_{2}=\left(\alpha_{i}^{(2)}\right) \in E$ with $\left\|x_{2}\right\|=1$ such that

$$
\left(3 K_{1}-1\right) / 4 \leqslant\||x|\|_{0} .
$$

Again, we may assume without any loss of generality that $\mathbb{N} \backslash\left(\sigma\left(x_{1}\right) \cup \sigma\left(x_{2}\right) \cup S_{1}\right)$ is infinite. Let $S_{2}$ be an infinite subset of $\mathbb{N} \backslash\left(\sigma\left(x_{1}\right) \cup \sigma\left(x_{2}\right) \cup S_{1}\right)$ such that the set $\mathbb{N} \backslash\left(\sigma\left(x_{1}\right) \cup \sigma\left(x_{2}\right) \cup S_{1} \cup S_{2}\right)$ is infinite and let $i_{2} \in \mathbb{N} \backslash\left(\sigma\left(x_{1}\right) \cup \sigma\left(x_{2}\right) \cup S_{1} \cup S_{2}\right)$. Writing

$$
F_{2}=\left\{y=\left(\beta_{i}\right) \in E:\left|\beta_{i_{2}}\right|=1, \quad \beta_{i}=\alpha_{i}^{(2)}, \text { for all } i \in \sigma\left(x_{1}\right) \cup \sigma\left(x_{2}\right) \cup S_{1} \cup S_{2}\right.
$$

and the set $\quad \mathbb{N} \backslash\left(\sigma(y) \cup \sigma\left(x_{1}\right) \cup \sigma\left(x_{2}\right) \cup S_{1} \cup S_{2}\right)$ is infinite $\}$

and $K_{2}=\sup \left\{\||y|\|_{0}: y \in F_{2}\right\}$, we have $\left\|2 x_{2}\right\|-1 \leqslant K_{2}$. This gives

$$
K_{2}-1 \leqslant\left((4 M-3) K_{1}-1\right) / 2 M \leqslant\left(K_{1}-1\right) / 2 \leqslant(M-1) / 4 .
$$

Continuing in this way, we get for each $n, x_{n}=\left(\alpha_{i}^{(n)}\right) \in E, S_{n} \subset \mathbb{N}, F_{n} \subset E$, a real number $K_{n}>1$ and a positive integer $i_{n}$ such that

(a) $x_{n} \in F_{n-1}, 1 \leqslant\left\|\mid x_{n}\right\|_{0} \leqslant K_{n-1}$ and $K_{n-1} \leqslant(M-1) / 2^{n}$, where $K_{0}=$ $M$, 
(b) if $M_{n}=\left(\bigcup_{j=1}^{n} \sigma\left(x_{j}\right)\right) \cup\left(\bigcup_{j=1}^{n-1} S_{j}\right)$, then $S_{n}$ is an infinite subset of $\mathbb{N} \backslash M_{n}$ such that $\mathbb{N} \backslash\left(M_{n} \cup S_{n}\right)$ is infinite,

(c) $i_{n} \in \mathbb{N} \backslash\left(M_{n} \cup S_{n}\right)$ and $\left|\alpha_{i_{n}}^{(n-1)}\right|=1$,

(d) $\alpha_{k}^{(n)}=\alpha_{k}^{(n-1)}$, for $k \in M_{n-1} \cup S_{n-1}$.

Thus, there is an $x_{0}=\left(\gamma_{i}\right) \in E$ such that

$$
\gamma_{k}=\left\{\begin{aligned}
\alpha_{k}^{(n)}, & \left(k \in M_{n} \cup S_{n}, \quad n=1,2, \ldots\right) \\
0, & \left(k \in \mathbb{N} \backslash\left(\bigcup_{n=1}^{\infty}\left(\sigma\left(x_{n}\right)\right) \cup S_{n}\right)\right)
\end{aligned}\right.
$$

Note that $\left|\gamma_{i_{n}}\right|=1,(n=1,2, \ldots)$ and consider the continuous linear functional on $E$ given by the Banach limit ([4], p.73) defined by

$$
f(y)=\operatorname{LIM}\left(\gamma_{i_{n}} \cdot \alpha_{i_{n}}\right), \quad\left(y=\left(\alpha_{i}\right) \in E\right) .
$$

Then, $f\left(x_{0}\right)=1$ and $f\left(x_{n}\right)=0,(n=1,2, \ldots)$.

Let for each $\lambda \in \Lambda, F_{\lambda}$ be a separable closed subspace of $G_{\lambda}$ such that

$$
\left\{x_{0}, x_{1}, \ldots\right\} \subset F=\overline{\operatorname{span}} \bigcup_{\lambda \in \Lambda} F_{\lambda} .
$$

Also, let $w_{\lambda}=v_{\lambda} \mid F$. Then, $\left(F_{\lambda}\right)_{\lambda \in \Lambda}$ is a strong $M$-decomposition of $F$ with the associated family of coordinate projections $\left(w_{\lambda}\right)_{\lambda \in \Lambda}$. For each $\lambda \in \Lambda$, let $\left(e_{n}^{(\lambda)}\right)_{n=1}^{\infty}$ be dense in $F_{\lambda}$. Write for each $n, U_{n}=\{A \subset \Lambda: \operatorname{card} A \leqslant n\}$ and $U=\bigcup_{n=1}^{\infty} U_{n}$. Let us define the following semi-norms on $F$

$$
\begin{aligned}
E_{A}^{(n)}(x) & =\inf \left\{\left\|x-\sum_{\lambda \in A} \beta_{\lambda} \sum_{i=1}^{n} \alpha_{i}^{(\lambda)} e_{i}^{(\lambda)}\right\|: \beta_{\lambda}, \alpha_{i}^{(\lambda)} \text { are scalars }\right\},(A \in U, n=1,2, \ldots) \\
F_{A}(x) & =\sum_{\lambda \in A} t_{\lambda}(x), \quad(A \in U), \\
G_{0}(x) & =\|x\|, \\
G_{n}(x) & =\sup \left\{E_{A}^{(n)}(x)+n F_{A}(x): A \in U\right\}, \quad(n=1,2, \ldots) .
\end{aligned}
$$

Define a mapping $Q: F \rightarrow c_{0}(\Delta)$ by

$$
(Q x)(\delta)=\left\{\begin{array}{l}
2^{-n} G_{n}(x), \quad(n=\delta / 2, \quad \delta=0,2,4, \ldots), \\
\alpha_{n} / n, \quad\left(n=(\delta+1) / 2, \quad \delta=1,3, \ldots \text { and } x=\left(\alpha_{i}\right)\right), \\
t_{\delta}(x), \quad(\delta \in \Lambda) .
\end{array}\right.
$$


Note that since $G_{n}(x) \leqslant\left(1+n^{2}\right)\|x\|,(n=1,2, \ldots)$, we have

$$
(Q x)(\delta) \leqslant\left(Q_{0} x\right)(\delta), \quad(x \in E, \delta \in \Lambda) .
$$

Thus, the function on $F$ given by

$$
\||x|\|=2 J(Q x)
$$

defines a norm on $F([11]$, Lemma) and, for each $x \in F$, satisfies

$$
\|x\| \leqslant\||x|\| \leqslant\||x|\|_{0}
$$

Finally, since $\left(\alpha_{n}\right) \rightarrow\left(\alpha_{n} / n\right)$ is an injective continuous linear operator of $E$ into $c_{0}$, that $\left(F_{\lambda}\right)_{\lambda \in \Lambda}$ is a strong $M$-decomposition of $F$ and that each $F_{\lambda}$ is separable, it follows (for example see the proof of Theorem 2 in [3], p.101) that the norm III.|\| is a locally uniformly convex on $F$. Now, note that for each $n$, the elements $x$ and $\left(x_{n}+x\right) / 2$ are in $F_{n}$. Therefore, $1 \leqslant\left\|\left|x_{n}\left\|\leqslant K_{n-1}, 1 \leqslant\right\|\right| x \mid\right\| \leqslant K_{n}$ and $2 \leqslant$ $\left\|\left|x_{n}+x\right|\right\| \leqslant 2 K_{n}$, where $K_{0}=M$ and for all $n$. This gives that $\||x|\|=\lim _{n \rightarrow \infty}\left\|\left|x_{n}\right|\right\|=$ $\lim _{n \rightarrow \infty} K_{n}=1$ and $\lim _{n \rightarrow \infty}\left\|\left|x_{n}+x\right|\right\|=2$. Hence $\lim _{n \rightarrow \infty} x_{n}=x$. But this is a contradiction and hence the proof is complete.

\section{REFERENCES}

[1] G.F. Bachelis and H.P. Rosenthal, 'On unconditionally converging series and biorthogonal systems in Banach spaces', Pacific J. Math. 37 (1971), 1-5.

[2] D.W. Dean, 'Schauder decompositions in (m)', Proc. Amer. Math. Soc. 18 (1967), 619-623.

[3] J. Diestel, Geometry of Banach spaces - selected topics: Lecture notes in Math. 485 (Springer-Verlag, Berlin, Heidelberg, New York, 1975).

[4] N. Dunford and J.T. Schwartz, Linear operators I, General Theory (Interscience, 1958).

[5] P.K. Jain and D.P. Sinha, 'On Markuševič decompositions of Banach spaces', Anal. Math. 16 (1990), 265-275.

[6] P.K. Jain and D.P. Sinha, 'On strong Markusevic decompositions of Banach spaces', $J$. Indian Math. Soc. 55 (1990), 117-126.

[7] J. Lindenstrauss, 'Weakly compact sets - their topological properties and the Banach spaces they generate', Proc. Sympos. on 'Infinite dimensional topology' held in Baton-Rouge, Ann. of Math. Studies 69 (1972), 235-274.

[8] J. Rainwater, 'Local uniform convexity of Day's norm on $c_{0}(\Gamma)$ ', Proc. Amer. Math. Soc. 22 (1969), 335-339.

[9] B.L. Sanders, 'On the existence of Schauder decompositions in Banach spaces', Proc. Amer. Math. Soc. 16 (1965), 987-990. 
[10] I. Singer, Bases in Banach spaces II (Springer-Verlag, Berlin, Heidelberg, New York, 1981).

[11] S. Troyanski, 'On locally uniformly convex and differentiable norms in certain non-separable Banach spaces', Studia Math. 37 (1971), 173-180.

Department of Mathematics

University of Delhi

Delhi-110007

India 